\title{
Formación en valores mediante la Investigación como Estrategia Pedagógica en la escuela
}

\section{Training in values through Research as a Pedagogical Strategy in school}

DOI: http://dx.doi.org/10.17981/cultedusoc.9.3.2018.97

Artículo de investigación. Fecha de recepción: 15/06/2018. Fecha de aceptación: 27/11/2018

\author{
Fray Garcia ${ }^{1}$; \\ Eunice Pertuz-Acevedo; Carolina Martínez-Pulido; \\ Deisy Luna-Muñoz; Emma González Aarón; Evelci Salas Mendoza; \\ Fanny Suarez Araujo; Graciela De la Cruz-Manga; Héctor Olaya; \\ Matilde Mejía Rodríguez; Mayerlis Taborda Cotes; Merly Pertuz Pérez; \\ Norma Lozano-Márquez; Nuris Puerto-Polo; Olga Oñate-Brujes; \\ Paola Pareja-Agamez y Silvana Chiquillo-Baldomino ${ }^{2}$ \\ Institución educativa Buenos Aires, sede San Martin (Colombia) \\ fraygarcia@hotmail.com
}

Para citar este artículo:

García, F., Pertuz-Acevedo, E., Martínez-Pulido, C., Luna-Muñoz, D., González, E., Salas, E., Suarez, F., De la Cruz-Manga, G., Olaya, H., Mejía, M., Taborda, M., Pertuz, M., Lozano-Márquez, N., Puerto-Polo, N., Oñate-Brujes, O., Pareja-Agamez, P. y Chiquillo-Baldomino, S. (2018). Formación en valores mediante la Investigación como Estrategia Pedagógica en la escuela. Cultura. Educación y Sociedad 9(3), 823-828. DOI: http://dx.doi.org/10.17981/cultedusoc.9.3.2018.97

\section{Resumen}

La educación ha presentado cambios significativos a lo largo del siglo XXI, donde el rol del docente y las estrategias pedagógicas que este utilice en el aula son elementos fundamentales que favorecen el proceso enseñanza - aprendizaje. En este artículo se buscó fomentar la formación en valores mediante la Investigación como Estrategia Pedagógica en educación básica. Se realizó un estudio de tipo descriptivo, de campo y transaccional, para escoger la muestra se ejecutó un muestreo por conveniencia donde se seleccionaron ciento ochenta (180) estudiantes de la IED Buenos Aires, sede San Martin en cursos comprendidos entre primero y quinto de primaria, donde se plantearon actividades de acuerdo al grado de escolaridad y edad. En la actualidad se evidencia dificultades a nivel de convivencias escolar en sus estudiantes, conflictos en el aula, que impiden el desarrollo oportuno de las clases, se observan con mayor frecuencia en la institución educativa Buenos Aires, por tal razón, se hace imperante diseñar un proyecto educativo encaminado a fomentar la formación de valores mediante la investigación como estrategia pedagógica IEP, para fomentar en los estudiantes relaciones efectivas, que aporten a la mejora de las relaciones interpersonales.

Palabras clave: Formación en valores, lúdica, investigación, educación básica.

\section{Abstract}

Education has presented significant changes during the 21 st century, where the role of the teacher and the teaching strategies that use this in the classroom are key elements that favour the process of teaching - learning. This article sought to promote training in values through research as a pedagogical strategy in basic education. A study of descriptive type, field and transactional, to choose the sample executed a sampling by convenience were selected where one hundred and eighty (180) students of the IED Buenos Aires, San Martin-based courses included between first and fifth grade, where activities according to the level of schooling and age were raised. Today there is evidence of difficulties at the level of school coexistence in its students, conflict in the classroom, that prevent the timely development of classes, are seen more frequently in school Buenos Aires, for this reason, it is prevailing design an educational project aimed at nurturing values through research as a pedagogical strategy IEP, to foster effective relationships, contributing to the improvement of interpersonal relationships in students.

Keywords: Training in values, play, research, basic education.

1 Líder del grupo de investigación "Capitanes de los valores".

2 Docentes de la institución educativa Buenos Aires, sede San Martin.

- The author; licensee Universidad de la Costa - CUC.

Cultura, Educación y Sociedad vol. 9 no. 3, pp. 823-828. Diciembre, 2018

Barranquilla. ISSN 2389-7724 Online 


\section{Introducción}

La educación en Colombia ha presentado avances significativos, permitiendo al docente implementar diversas metodologías innovadoras fortaleciendo el proceso enseñanza-aprendizaje. Actualmente los casos de acoso escolar o Bullying se observan con frecuencia en la institución educativa Buenos Aires, por tal razón se hace imperante diseñar estrategias pedagógicas que apunten al desarrollo de competencias del ser a través de la formación en valores.

Formar en valores implica, educar al estudiante para que encuentre sentido en sus acciones, responsabilizándose por las mismas, lo cual le permitirá tomar decisiones asertivas, desarrollar capacidades como solución de conflictos, pensamiento crítico, que a su vez le posibilite plantear objetivos, para buscar la manera más idónea de abordar la realidad, presentándole al educando una formación con una dimensión humana e integral, que permita el desarrollo libre de su personalidad, partiendo de la comprensión de los derechos del otro, (Conejo, 2012; Ramírez y Hugueth (2017); Herrera, Guerrero y Ramírez, 2018).

Orientados al aporte de una educación integral el cuerpo docente de la IED Buenos Aires, sede San Martin, identificó dificultades a nivel de convivencia escolar en sus estudiantes, observando conflictos en el aula, que impedían el desarrollo oportuno de las clases, por lo cual surgió la necesidad de diseñar un proyecto educativo encaminado a fomentar la formación en valores mediante la Investigación como Estrategia Pedagógica (IEP) en la escuela, para fomentar en los estudiantes relaciones afectivas, que aporten a la mejora de relaciones interpersonales, estableciendo pautas elementales de convivencia para aplicar dentro y fuera del aula, donde se diseñaron actividades articuladas a los lineamientos curriculares y el plan de área, implementando la investigación para lograr aprendizajes significativos.
Elementos conceptuales de la formación en valores mediante la IEP en la escuela

Actualmente la formación en valores es una de las temáticas más estudiadas en el debate educativo, debido al deterioro en materia de conciencia ciudadana observado en todos los niveles sociales, evidenciando conductas como; agresiones entre grupos étnicos, discriminación, manifestación de inconformidad social, diversas formas de violencia real y simbólica en el trato entre personas, familiares y compañeros en el ámbito escolar. Por lo anterior la escuela mediante proyectos curriculares ha intentado impulsar la formación en valores, no obstante, se convierte en un reto para la educación básica, donde se entiende que este tipo de enseñanza debe estructurarse más allá de planteamientos teóricos (Díaz, 2006).

Según Arufe, (2011) y Ramírez, Avendaño, Aleman, Lizarazo, Ramírez y Cardona (2018), los valores se constituyen y se aprenden fundamentalmente en la infancia y la adolescencia, a partir de los modelos sociales interiorizados en el núcleo familiar, la educación impartida por los docentes en el ámbito escolar, la información observada en medios de comunicación y la interacción con su grupo de pares, por lo cual la escuela debe tener en cuenta la influencia de estos factores en la formación ética del estudiante, para diseñar estrategias pedagógicas que vinculen la esfera afectiva y las competencias del ser.

Por consiguiente, contener dentro de la malla curricular la asignatura de ética y valores, no es garantía de realizar un proceso de formación en valores adecuado que presente un impacto positivo en la comunidad educativa, puesto que se hace necesario transversalizar dicha área a las diferentes asignaturas integrándola al proceso de enseñanza - aprendizaje, esto se logra cuando los estudiantes se conviertan en individuos capaces de entender y transformar la realidad, adquiriendo conocimientos, pero a la vez, analizando a fondo los fenómenos que suceden en la vida cotidiana, donde el es- 
tudiante debe asumir una actitud crítica y constructiva en favor del desarrollo de los valores éticos básicos para la vida y la convivencia social que fundamentan el sistema democrático. Para Botero (2006) citado por Ramírez, Chacón y El Kadi (2018), si el proceso se realiza de esta manera, la institución conseguirá el desarrollo de una educación integral humanizadora

En ese sentido, para formar ciudadanos integrales, con competencias del ser es necesario diseñar estrategias pedagógicas basadas en valores, que permitan a los estudiantes relacionarse con los demás y adquirir progresivamente pautas básicas de convivencia, necesarias para relacionarse en sociedad, así como prepararse para la solución pacífica de conflictos y para el ejercicio activo de la ciudadanía, siendo tolerante frente las diferencias, respetando los derechos humanos y el pluralismo propio de una sociedad democrática (Conejo, 2012; Ramírez, Chacón y Valencia, 2018).

Partiendo de las consideraciones anteriores, se hace hincapié en la noción de comprender que los valores no pueden ser enseñados de la misma forma como se enseñan los contenidos curriculares, puesto que se debe realizar una interiorización de los mismos, este proceso implica tres componentes; el cognitivo, que hace referencia a los conocimientos, el componente afectivo; que implica los sentimientos del individuo y el componente conductual o conativo; que incluye las acciones manifiestas, sin embargo, cada persona, debe construir su propio esquema de valores, donde la función de los educadores es colaborar en el proceso, permitiendo desarrollar situaciones en el entorno de los alumnos para que los experimenten y de esta manera puedan ser interiorizados por los mismos (Parra, 2003; Ramírez y Ampudia, 2018).

En ese sentido, para fortalecer los valores escolares es necesario implementar estrategias pedagógicas innovadoras que transformen los modelos educativos tradicionales, esto es posible mediante metodologías basadas en investigación, estas hacen referencia a un proceso sistemático y organizado que tiene como propósito responder a una pregunta, lo cual permite aumentar los conocimientos y conocer información sobre algo desconocido (Vital, 2009).

Los valores éticos, dicen Sisiruca y Salazar (2014), juegan un papel fundamental, porque forman parte importante de todas las actividades que se realiza el ser humano dentro de los patrones de cooperación para todos los proyectos que quiere emprender en la vida. Además, en el contexto de la importancia de la eticidad, los autores Núñez y Ravina (2017) expresan que la UNESCO quiere que los países establezcan un marco ético y reglamentario destinado a la protección jurídica en las investigaciones y otros.

En ese sentido la investigación es utilizada para realizar actividades intelectuales y experimentales con el propósito de conocer de un tema determinado. Para integrarla a los procesos educativos Mejía y Manjarrés, (2010). Proponen la investigación como estrategia pedagógica (IEP), esta es concebida como una metodología educativa que permite integrar el aprendizaje en contexto mediante la investigación tomando elementos de la pedagogía critico-liberadora y del enfoque socio-cultural.

Según Pino y Urrego, (2013) la formación integral de seres humanos es uno de los pilares básicos de la educación, integrando componentes cognitivos y sociales que permitan el desarrollo pleno de los sujetos, mediante la construcción de habilidades y competencias personales que favorezcan la creación de un proyecto de vida, pero, además, posibilite el desempeño en la sociedad como seres democráticos y ciudadanos, que respeten los derechos de los demás.

Desde la metodología propuesta por la IEP los contenidos curriculares se convierten en problemas, donde el docente a partir de las preguntas planteadas por los estudiantes diseña unas trayectorias de indagación, así, como avances y desarrollo por parte de los estudiantes en el que se deter- 
minan una ampliación de la estructura del lenguaje, en donde se manifiesta un conocimiento solido en pertinencia a un léxico coherente orientado por facilitador, quien se encargara de la planificación de los ejercicios y los métodos con el fin de cumplir los objetivos trazados para tal fin (Pozo, 2006).

Por lo anterior, la propuesta de la IEP es construir una cultura ciudadana y democrática en ciencia, tecnología e innovación. Este ejercicio pedagógico aparece como fundamento de una nueva forma de lo público que, a través de la idea de justicia educativa y justicia curricular, trabaja por construir sociedades más justas y menos desiguales. La propuesta metodológica se fundamenta en valores que buscan desarrollar capacidades de cognitivas, afectivas, valorativas y de acción (Ortega, 2009).

En resumen, el docente desde la IEP parte de la pregunta de sentido común de los estudiantes, la redirecciona a la perspectiva de la educación, reconociendo la existencia de saberes comunes o elaborados, asociando los conocimientos disciplinares con las experiencias socioculturales, promoviendo así la apropiación practica desde el pensamiento crítico buscando transformar contextos, culturas, epistemologías, y redirigir sus escenarios de poder para construir subjetividades y ciudadanías (Mariño, 2010).

\section{Metodología}

Se realizó una investigación de tipo descriptivo, de campo y transeccional, puesto que esta buscó describir el proceso de formación en valores a través de la investigación como estrategia pedagógica, desde un trabajo de campo, donde se establecieron actividades para alcanzar dicho objetivo, en un tiempo y espacio establecido. Los estudios con diseño descriptivo están encaminados a especificar las características, perfiles y propiedades de personas, grupos, comunidades, procesos, objetos o cualquier otro fenómeno que sea sometido a un análisis. (Hernández, Fernández y Baptista, 2010).
Por su parte, Sabino (2006), indica que las investigaciones de campo, permiten que el investigador recolecte los datos de forma directa con la realidad o el objeto de estudio, lo cual permite obtener un conocimiento profundo sobre el problema a investigar. Además, los estudios con diseño transeccional, buscan que los datos se recolecten en un momento y tiempo determinado. Gómez (2006), establece que el diseño transeccional recolecta datos en un momento y tiempo único, donde su objetivo es describir variables y analizar su incidencia en un momento especifico.

La población beneficiaria con este estudio corresponde a la Institución Educativa Departamental Buenos Aires localizada en el corregimiento de Sampués, ubicado en Aracataca, Magdalena, la sede San Martin se encuentra en la parte urbana cerca del límite con el Municipio de Fundación, para escoger la muestra se realizó un muestreo por conveniencia donde se seleccionaron ciento ochenta (180) estudiantes de la IED Buenos Aires, sede San Martin en cursos comprendidos entre primero y quinto de primaria, donde se plantearon actividades de acuerdo al grado de escolaridad y edad.

\section{Resultados}

Esta investigación pretende propiciar en el lector conexiones entre los sustentos teóricos de la formación en los valores mediante la investigación como estrategia pedagógica. En la actualidad se evidencia dificultades a nivel de convivencia escolar en sus estudiantes, observando conflictos en el aula, que impedían el desarrollo oportuno de las clases, por lo cual surgió la necesidad de diseñar un proyecto educativo encaminado a fomentar la formación en valores mediante la Investigación como Estrategia Pedagógica (IEP) en la escuela, para fomentar en los estudiantes relaciones afectivas, que aporten a la mejora de relaciones interpersonales, estableciendo pautas elementales de convivencia para aplicar dentro y fuera 
del aula, donde se diseñaron actividades articuladas a los lineamientos curriculares y el plan de área, implementando la investigación para lograr aprendizajes significativos.

Muchas de estas inquietudes son acompañadas de un modo generalizado, en el que la identidad por la usencia en un sentido espontaneo coherente y claro en cuanto a la pertinencia, están dado por la carencia de proyectos de vida que estén enfocados en unificar la fe, la armonía de todos los actores en pertinencia, esto mediante la capacidad de crear cambios cónsonos en todos los involucrados, por la supremacía del conocimiento y la razón, que expresen racionalidad instrumental-administrativagerencial, capaz de aplastar lo afectivo y consolidar lo referente a la parte sentimental.

\section{Conclusiones}

Los hallazgos encontrados confirman que hoy por hoy vivimos en una sociedad sin criterios de valores, paradigmas socioeconómicos y cultural, lo que a la postre produce confusión y desorientación en la sociedad, y sobre todo estos son llevados a la las comunidades educativas, en ese sentido, es de suma importancia poder abordar dicho asunto teniendo como base todos los actores de la sociedad que sirvan de conductores en la búsqueda de una salida ante esta situación, ello requiere de un compromiso de todos y para todos, que sirva de guía para enfrentar los propios retos en proporción del desarrollo científico, y globalizarlo para los cambios integrales que requiere urgentemente en todos los sentidos.

En ese mismo sentido, la realidad es que en las instituciones educativas ha venido cayendo la puesta en práctica de los principios más importante para un buen vivir, como son los valores, la tolerancia, el buen trato con los estudiantes, los docentes, la comunidad, en fin, todos los actores que hacen parte de esta sociedad tan exigente hoy en día. Cabe decir, el mismo busca la transfor- mación mediante la investigación en todos los niveles. En ese sentido, el docente como actor principal en el aula de clases debe desarrollar actividades llenas de creatividad para poder contrarrestar este flagelo de la intolerancia, con un propósito no solo en conceptos o contenidos, sino que enriquezcan la dimensión social y moral, es decir en la práctica y en el hacer. Ya que hoy en día enseñar los valores desde la practicidad es más significativo en el niño y el resto de la sociedad además de contribuir en el mejoramiento de la calidad de convivencia

\section{Referencias}

Arufe, V. (2011). La educación en valores en el aula de educación física. ¿mito o realidad? Revista Digital de Educación Fisica, 2(9).32-42. Recuperado de https:// dialnet.unirioja.es/servlet/ articulo?codigo $=3618413$

Botero, C. (2006). Los ejes transversales como instrumento pedagógico para la formación en valores. Revista Politécnica, 2(3). 49-59. Recuperado de http://132.248.9.34/hevila/Revistapolitecnica/2006/no3/5.pdf

Conejo, P. (2012). El valor formativo de la música para la educación en valores. Revista de Educação e Humanidades, 2. 263-278. Recuperado de http://dialnet. u n i r i o j a e s / s e r v l e t / oaiart?codigo $=3825651$

Díaz, A. (2006). La educación en valores: Avatares del currículum formal, oculto y los temas transversales. Revista electrónica de investigación educativa, 8(1).

Galrin, J. y Fernández, J. (2010). Enseñar matemáticas con recursos de ajedrez. Revista. Tendencias Pedagógicas, 1(15). 57-90. Recuperado de https://revistas. uam.es/tendenciaspedagogicas/article/ view/1933/2043

Gómez, M. (2006). Introducción a la metodología de la investigación científica. España: Editorial brujas. 
Herrera, B., Guerrero, H. y Ramírez, R. (2018). Investigación como estrategia pedagógica: Una mirada desde la educación, escuela y transformación de la comunidad global. Barranquilla: Editorial Universitaria de la Costa.

Hernández, R., Fernández, C. y Baptista, M. (2010). Metodología de la Investigación (5ta ed.). México, D.F.:.The MacGraw-Hill.

Landinez-Lamadrid, D., Ramirez-Ríos, D., Neira, D., Parra, K. y Combita, J. (2017). Shapley Value: its algorithms and application to supply chains. INGE CUC, 13(1), 61-69 https://doi.org/10.17981/ingecuc.13.1.2017.06

Mariño, G. (2010). El diálogo en la educación de jóvenes y adultos. Dos propuestas pedagógicas para implementarlo. En, El taller dialógico / la recuperación de experiencias laborales. Bogotá. OEI.

Mejía, M. y Manjarrés, M. (2010) La Investigación como Estrategia Pedagógica. Bogotá, D.C.: Programa Ondas - Colciencias.

Núñez, E. y Ravina, R. (2017). Análisis del nivel de competitividad empresarial en el panorama publicitario colombiano fundamentada en el éxito creativo y la responsabilidad ética y jurídica. Juridicas CUC, 13(1), 9-28.

Ortega, P., Peñuela, D. y López, D. (2009). Sujetos y prácticas de la pedagogía crítica. Ediciones El Búho. Bogotá.

Parra, J. (2003). La Educación en valores y su práctica en el aula. Revista Tendencias Pedagógicas, 8. 66-69.

Pino, M. y Urrego, Y. (2013). La importancia de las funciones ejecutivas para el desarrollo de las competencias ciudadanas en el contexto educativo. Cultura Educación y Sociedad, 4(1).

Pozo, J. y Gómez, M. (2006). Aprender y enseñar ciencia. 5ta. Edición. Madrid: Ediciones Morata.
Ramírez, R. y Hugueth, A. (2017). Modelo de comunicación productiva para las organizaciones de salud pública en Venezuela. Revista Opción. 33(83). 305-335. Disponible en http://produccioncientificaluz.org/index.php/opcion/article/ view/23118

Ramírez, R. y Ampudia, D. (2018). Factores de Competitividad Empresarial en el Sector Comercial. Revista Electrónica de Ciencia y Tecnología del Instituto Universitario de Tecnología de Maracaibo (RECITIUTM). 4(1). 16-32. Disponible en http://recitiutm.iutm.edu.ve/ index.php/recitiutm/article/view/130

Ramírez, R., Avendaño, I., Aleman, L., Lizarazo, C., Ramírez, R. y Cardona, Y. (2018). Principles of social responsibility for the strategic management of the talent human public health organizations. Revista Espacios, 39(37). 22-27. Disponible en http://www.revistaespacios.com/a18v39n37/18393722.html

Ramírez, R., Chacón, H. y Valencia, K. (2018). Gestión del talento humano como estrategia organizacional en las Pequeñas y Medianas Empresas. Revista CICAG, 16(1): 20-42. Disponible en http:// ojs.urbe.edu/index.php/cicag/article/ view/2929

Ramírez, R., Chacón, H. y El Kadi, O. (2018). Gestión estratégica del talento humano en las PYMES. Medellín: Editorial Corporación CIMTED.

Sabino, C. (2006). Como hacer una tesis y elaborar todo tipo de escritos. Venezuela: Editorial Panapo.

Sisiruca, M. y Salazar, C. (2014). Valores éticos de la responsabilidad social interna en centros de producción audiovisual. Económicas CUC, 35(1), 79-90.

Vital, M. (2009). La investigación en los procesos de enseñanza aprendizaje. Rev. Vida Científica, 6. 